Ann. Abeille, Ig63, 6 (3), 229-234.

\title{
OBSERVATIONS PRÉLIMINAIRES SUR LA RÉCOLTE DU POLLEN PAR LES ABEILLES DANS “ LES GRANDES LANDES "DE LA FORÊT LANDAISE
}

\author{
J. LOUVEAUX et J. ALBISETTI
}

Station de Recherches sur l'Abeille et les Insectes sociaux, Bures-sur-Yvette (Seine et Oise)

\section{SOMMAIRE}

Les auteurs ont étudié la récolte du pollen par les abeilles dans " les Grandes Landes " de la forêt landaise, au cours de l'été I962. Les observations ont été faites en utilisant la technique habituelle des trappes à pollen.

Du point de vue quantitatif, des différences très importantes ont été notées entre les ruches sédentaires et les ruches amenées en transhumance. Par contre, en raison de l'extrême pauvreté variétale de la flore, on ne note que des différences qualitatives mineures.

On discute de l'importance des résultats obtenus sous l'angle de la nutrition et de l'état sanitaire des colonies.

\section{INTRODUCTION}

De juin à novembre, la Forêt landaise offre aux abeilles une floraison à peu près continue d'Ericacées (Evica tetralix, Erica cinerea, Calluna vulgaris) qui justifie un vaste mouvement de transhumance des ruches des régions voisines en vue de l'utilisation d'une miellée généralement abondante.

Le miel de Callune trouve sur le marché nord-européen des débouchés importants à des prix très rémunérateurs, si bien que sa production mérite des recherches particulières qui viennent d'être entreprises dans le cadre de 1'I. N. R. A. Ces recherches devront se faire dans différentes directions mais ce sont certainement les problèmes de pathologie du couvain qui nécessitent les études les plus urgentes. Certes, 1a pharmacopée moderne permet dans la pratique de lutter efficacement 
contre les Loques. Toutefois, si l'on tient compte de l'incidence économique des traitements sur le prix de revient du miel (temps passé, surveillance étroite et sans relâche des colonies), il est évident qu'il serait du plus haut intérêt de connaître avec précision quels sont les facteurs d'ordre écologique qui sont susceptibles de favoriser dans la Lande l'extension des maladies du couvain. Parmi ces facteurs, la première place revient sans doute aux conditions d'alimentation des colonies. Il est en effet possible, sinon probable, qu'il existe un lien entre le développement des maladies du couvain et l'alimentation des abeilles, mais rien ne permet actuellement d'être affirmatif à ce sujet.

C'est en partant de ces considérations qu'il nous est apparu que, dans un premier stade, il serait particulièrement intéressant de pouvoir dresser un tableau complet des conditions rencontrées par l'abeille landaise pour son alimentation. Dans ce travail purement préliminaire, nous exposons le résultat de quelques observations susceptibles de contribuer à une meilleure connaissance des conditions d'existence des colonies d'abeilles au sein de la flore si particulière des Landes de Gascogne.

\section{MATÉRIEL, E'T MÉTHODE}

Les observations ont été conduites à Sabres (Landes) du 28 juillet r962 jusqu'au début de novembre de la même année. Le rucher expérimental se trouve en pleine forêt de Pins maritimes. Des landes de différents types constituent l'aire de butinage des abeilles; ce sont des landes sèches assez riches à Erica cinerea, Calluna vulgaris et Ulex europaeus, des landes sèches assez pauvres à Calluna vulgaris et Erica scoparia, des landes drainées assez riches à Pteris aquilina et Ulex europaeus, des landes humides assez riches à Molini cerulea et Ulex namus. Notons encore que Rhamnus frangula est abondant dans le sous-bois.

TABLEAU I

\begin{tabular}{|c|c|c|c|c|}
\hline & $\begin{array}{l}\text { Température } \\
\text { moyenne }\end{array}$ & $\begin{array}{l}\text { Écart à la } \\
\text { normale }\end{array}$ & $\begin{array}{l}\text { pluviom. } \\
\text { (en mm) }\end{array}$ & $\begin{array}{c}\text { Écart à la } \\
\text { normale (mri) }\end{array}$ \\
\hline Janvier $\ldots \ldots \ldots \ldots \ldots \ldots \ldots \ldots \ldots$ & $7^{\circ} 0$ & $1^{\circ} 4$ & 81 & -4 \\
\hline 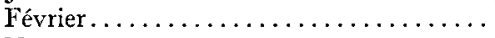 & $5^{\circ} 6$ & -100 & 52 & -28 \\
\hline $\operatorname{Mars} \ldots \ldots \ldots \ldots \ldots \ldots \ldots \ldots \ldots$ & $7 \circ 1$ & -202 & 68 & $\longrightarrow 2$ \\
\hline Avril. . . . . . . . . . . . . . . . . & 1103 & $-0^{\circ} 7$ & 70 & -10 \\
\hline Mai........................ & 1403 & -007 & 75 & -10 \\
\hline Juin $\ldots \ldots \ldots \ldots \ldots \ldots \ldots \ldots$ & $18^{\circ} 1$ & $-0^{\circ} 6$ & 18 & -48 \\
\hline Juillet $\ldots \ldots \ldots \ldots \ldots \ldots \ldots \ldots$ & $20^{\circ} 3$ & -003 & 13 & -42 \\
\hline Août $\ldots \ldots \ldots \ldots \ldots \ldots \ldots \ldots$ & $21^{\circ} 1$ & $0 \circ 7$ & 16 & -54 \\
\hline Septembre $\ldots \ldots \ldots \ldots \ldots \ldots \ldots$ & $18^{\circ} 7$ & 007 & 40 & $\longrightarrow 30$ \\
\hline Octobre $\ldots \ldots \ldots \ldots \ldots \ldots \ldots$ & 1407 & 103 & 59 & -17 \\
\hline 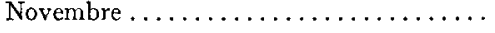 & $6^{\circ} 3$ & -205 & 94 & 一 6 \\
\hline
\end{tabular}

Au 28 juillet, la trappe à pollen a été posée sur cinq ruches constituant deux lots, l'une des des trois colonies transhumantes, l'autres de deux colonies sédentaires. Ces colonies de force très sensiblement équivalente, étaient de même race, à savoir la race locale landaise. Les ruches $\mathrm{n}^{\mathbf{0}} 35$ et $n^{0} 44$ étaient deux ruches sédentaires n'ayant jamais quitté leur emplacement de Sobres. Par contre les ruches $\mathrm{A}, \mathrm{B}$ et $\mathrm{Z}$ venaient de Chalosse où elles avaient passé tout le début de l'année

Une fois les trappes mises en place, les récoltes de pollen ont été faites régulièrement une ou deux fois par semaine. Le pollen était séché à l'étuve à $40^{\circ}$, puis pesé à un gramme près. 
L'analyse qualitative a été faite à Bure-sur-Yvette selon les techniques déja décrites par l'un de nous (LOUVEAUX, 1958).

Unc ruche sur bascule placée à proximité immédiate du rucher expérimental nous a fourni des indications précises sur l'évolution de la miellée.

Une visite mensuelle de toutes les ruches nous a fourni des éléments d'appréciation de l'évolution des surfaces de couvain, de miel et de réserves de pollen.

Ne disposant pas d'un poste météorologique, nous n'avons pas pu procéder à des relevés de température ou de pluviométrie. Nous empruntons au Bulletin mensuel de la météorologie nationale les données générales concernant les conditions climatiques de l'été 1962 dans les landes (Poste d'observation de Mont-de-Marsan) (tableau r.)

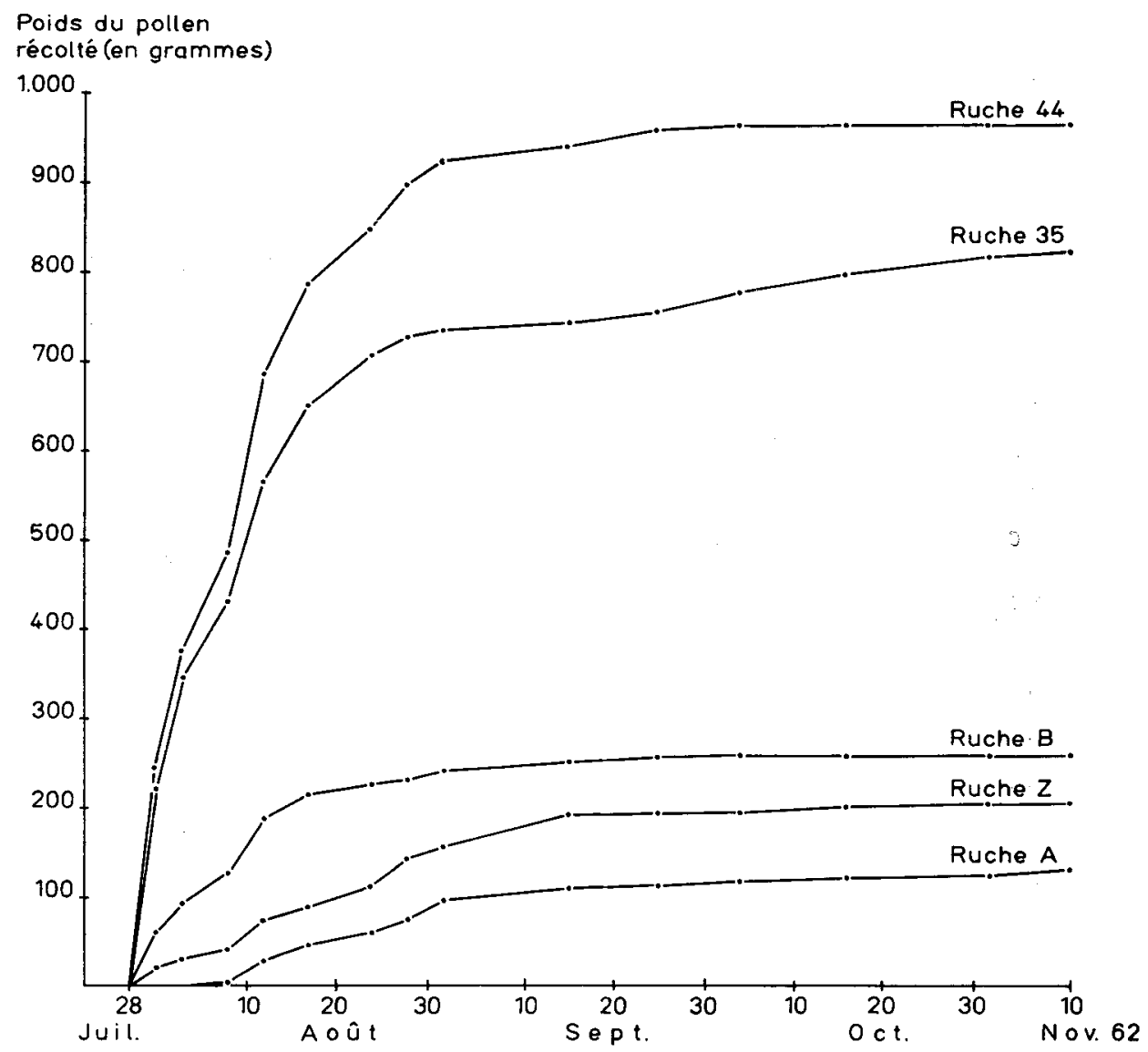

FIG. I. - Courbes cumulatives du poids du pollen récolté de juillet par les différentes ruches mises en observation

\section{RÉSULTATS}

$\mathrm{I}^{\mathrm{O})}$ Résultats quantitatifs.

La figure I regroupe les données quantitatives recueillies sous forme de courbes cumulatives. En gros, on constate d'importantes récoltes de pollen jusque vers le I5 septembre puis des récoltes minimes jusqu'au ro novembre.

Annales de l'Abeille. - 1963 . 

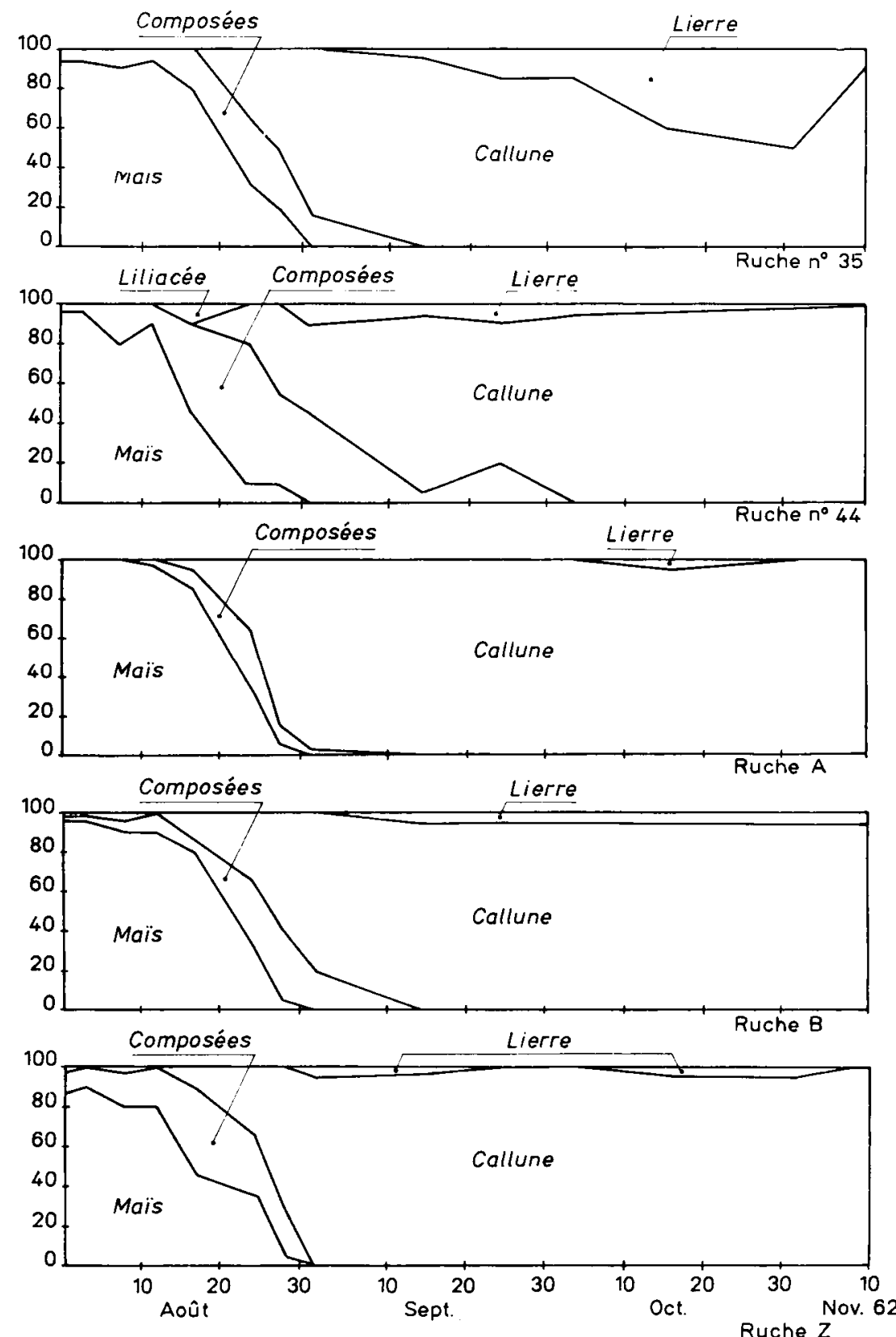

Fig. 2. - Composition des récoltes de pollen des différentes ruches mises en observation

Io novembre. Il est particulièrement intéressant de noter que les ruches sédentaires ont fait des récoltes beaucoup plus importantes que les ruches transhumantes (Nous discuterons plus loin les raisons apparentes de ce phénoméne). Toutefois, l'allure 
générale des courbes de récolte est peu différente d'une ruche à l'autre; seul le taux de récolte varie.

\section{$\left.2^{\circ}\right)$ Résultats quantitatifs.}

L'examen qualitatif des récoltes met en évidence deux phénoménes (fig. 2). Le premier est l'extrême pauvreté en espèces visitées; le second est la relative uniformité des récoltes d'une ruche à l'autre. En juillet et en août, on peut dire que le Maïs constitue la seule source de pollen ou à peut près. Il est progressivement remplacé par la Callune qui fournit ensuite la presque totalité de pollen récolté, le lierre mis à part.

\section{DISCUSSION}

Il convient avant tout de mettre bien en évidence le caractère assez anormal de l'année I962 du point de vue climatique. Le tableau I met en évidence un déficit pluviométrique important et des températures moyennes au-dessus de la normale pendant toute la période considérée. Dès le mois d'août, la lande a pris un aspect brûlé et les colonies ont cessé de trouver du nectar dans les fleurs ainsi qu'en témoi-

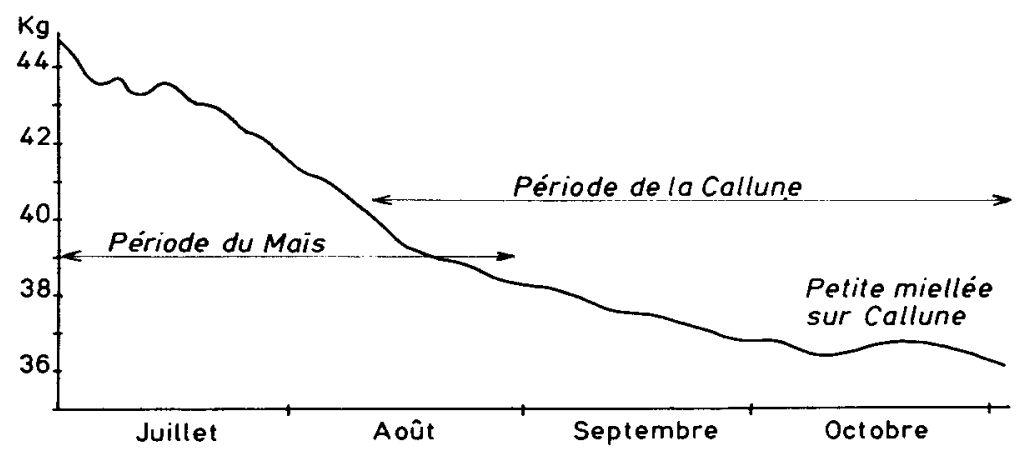

FIG. 3. - Variations de poids d'une ruche sur bascule entre juillet et octobre ig62 à Sabres (Landes)

gne la courbe de poids (fig. 3) de la ruche sur bascule. L'étendue du couvain a été en baisse constante et régulière dans toutes les ruches de juillet à novembre. De ro à I 2 cadres de couvain en juillet, les colonies sont retombées à 7 cadres aul I5 aotit et à 3 cadres au 20 septembre.

Pour toutes ces raisons, on ne peut donc pas considérer l'année I962 comme entièrement normale, surtout à partir du $\mathrm{I}_{5}$ août où le déficit pluviométrique s'est fait particulièrement sentir. Cette réserve faite, on ne peut pas ne pas être frappé par le caractère vraiment spécial de l'approvisionnement des ruches en pollen dans la Lande. Les seuls pollens accessibles à partir de juillet sont des pollens considérés généralement comme peu attractifs pour les abeilles. On sait par expérience que le Maïs n'est visité avec intensité qu'en période de disette et lorsqu'aucune autre plante n'offre un pollen plus attractif. Il en va de même pour la Callune dont le pollen n'est pour ainsi dire pas récolté en dehors des zones où sa floraison représente la seule source de pollen accessible aux abeilles. On note par contre l'absence totale 
de pollens de Papillionacées, Rosacées et Crucifères qui constituent d'ordinaire un élément essentiel de l'alimentation des abeilles.

Le fait que les colonies transhumantes aient récolté beaucoup moins de pollen que les colonies sédentaires s'explique par le fait que les ruches venues de Chalosse étaient abondamment approvisionnées en pollen alors que les ruches sédentaires n'avaient pas de réserves. En fin juillet, il y a deux cadres pleins de pollen dans les ruches A, B et $Z$. Par contre, les ruches 35 et 44 n'ont pratiquement pas de pollen stocké et vivent au jour le jour. On est donc amené à penser que les pollens offerts par la lande étant dans leur grande majorité des pollens peu attractifs, les abeilles transhumantes ont vécu sur leurs réserves. Au contraire, les ruches sédentaires se trouvant en état de disette, ont dû s'approvisionner de force sur les seules sources existantes, quelle que soit leur valeur alimentaire.

Il serait prématuré d'établir un lien direct entre le caractère anormal des récoltes de pollen dans la lande et le développement des maladies du couvain mais on ne peut s'empêcher de penser qu'une alimentation aussi déséquilibrée que celle que nous avons mis en évidence ne peut que contribuer à diminuer la résistance des larves aux attaques microbiennes.

Reçu pour publication en mai 1963.

\author{
SUMMARY \\ PRELIMINARY OBSERVATIONS ON POLLEN GATHERING BY BEES IN THE " GRANDES LANDES" \\ OF THE LANDES FOREST
}

The authors have studied pollen gathering by bees in the "Grandes Landes " of the Landes forest, during summer 1962. They have used the usual method of pollen-traps.

Important quantitative differences were noted between fixed and moved hives. On the other hand, only slight qualitative differences were noted, due to the small number of varieties of the flora.

'The importance of these results is discussed as regards nutrition and health of the colonies.

\title{
RÉFÉRENCE，BIBLIOGRAPHIQUE
}

Lotveaux J. 1958. Recherches sur la récolte du pollen par les abeilles. Thèse, 206. p. 